\title{
Antidepressant Mechanism Research of Acupuncture: Insights from a Genome-Wide Transcriptome Analysis of Frontal Cortex in Rats with Chronic Restraint Stress
}

\author{
Yu Wang, ${ }^{1}$ Huili Jiang, ${ }^{1}$ Hong Meng, ${ }^{2}$ Jing Li, ${ }^{1}$ XinJing Yang, ${ }^{1}$ Bingcong Zhao, ${ }^{1}$ \\ Yang Sun, ${ }^{1}$ and Tuya Bao ${ }^{1}$ \\ ${ }^{1}$ School of Acupuncture, Moxibustion and Tuina, Beijing University of Chinese Medicine, Beijing 100029, China \\ ${ }^{2}$ School of Science, Beijing Technology and Business University, Beijing 100048, China \\ Correspondence should be addressed to Tuya Bao; tuyab@263.net
}

Received 5 May 2017; Revised 12 July 2017; Accepted 26 July 2017; Published 26 September 2017

Academic Editor: Gang Chen

Copyright @ 2017 Yu Wang et al. This is an open access article distributed under the Creative Commons Attribution License, which permits unrestricted use, distribution, and reproduction in any medium, provided the original work is properly cited.

\begin{abstract}
Major depressive disorder (MDD) is a chronic disease that adversely affects mood and cognition. In this study, we randomly divided the rats into control group (C), model group (M), fluoxetine group (F), and acupuncture group (A), used open-field test to ascertain whether acupuncture affects chronic restraint stress (CRS) induced depression-like behaviors of rats, and explored the antidepressant mechanism of acupuncture at the molecular level of transcriptome in the frontal cortex of CRS rats by RNAsequencing (RNA-seq). According to differentially expressed genes (DEG) analysis, we identified 134, 46, and 89 response genes differentially expressed in $C$ versus $M, F$ versus $M$, and A versus $M$, respectively. Through Gene Ontology (GO) term enrichment analysis and Kyoto Encyclopedia of Genes and Genomes (KEGG) pathway enrichment analysis, we identified the gene sets involved in extracellular space, inflammatory response, Toll-like receptor signaling pathway, chemokine signaling pathway, and TNF signaling pathway. In this study, RNA-seq technology was used to investigate the frontal cortex genome-wide transcriptomes in depression rats under CRS, which suggested that the antidepressant effect of acupuncture is effective and has a multitarget characteristic, which may be related to amino acid metabolism and inflammatory pathways, especially the Toll-like receptor signaling pathway, TNF signaling pathway, and NF-kappa B signaling pathway.
\end{abstract}

\section{Introduction}

Major depressive disorder (MDD) is a chronic disease that adversely affects mood and cognition [1]. Morbidity and mortality associated with MDD have a significant effect on individuals with the illness and their families. A variety of brain imaging, postmortem morphometric, and other studies have shown that MDD is involved in the regulation of emotional and cognitive brain regions, such as the frontal cortex and hippocampus [2-4]. In spite of the fact that there is considerable evidence shown that the defects of multiple genes are associated with genetic susceptibility to MDD [5], the molecular basis has been elusive.

Nowadays, in the treatment of depression, it is estimated that approximately $30-50 \%$ of depressive patients have no response to the approved antidepressant treatment, which is thought to reflect a lack of specificity in targeting underlying pathological mechanism of depression [6,7]. As an important part of Chinese medicine, acupuncture is an effective complement and alternative to antidepressant therapy [8]. So far, numerous studies have been published in the field of depression and acupuncture; most of them hold a positive attitude towards the antidepressant efficacy of acupuncture [9-13]. However, what is the molecular mechanism of acupuncture antidepressant effect?

Many studies have shown that a variety of molecular and intracellular signal transduction pathways that are closely related to inflammation and immunity are involved in the pathogenesis of depression $[7,14,15]$. In previous studies, we also found that acupuncture could significantly reverse the depression-like behavior of rats induced by chronic stress and have an effect on the molecular and intracellular signal 
transduction pathways that are closely related to inflammation and immunity [16-20], suggesting that acupuncture may play a role in the regulation of multiple targets in depression. Then, is there also a special mechanism for acupuncture antidepressant?

Therefore, in this study, we used open-field test to ascertain whether acupuncture affects CRS induced depressionlike behaviors in rats, and explore the antidepressant mechanism of acupuncture at the molecular level of transcriptome in the frontal cortex of CRS rats by RNA-seq.

\section{Materials and Methods}

2.1. Animals. Forty-eight male Sprague-Dawley (SD) rats $(180 \pm 20 \mathrm{~g})$ were used in this study. All animals were obtained from Charles River Laboratories of Beijing, China (license number SCXK (Jing) 2012-0001). In terms of the living conditions for the rats, they were housed in the cages with free access to water and food under the circadian of 12-hour light/dark (light on at 8:00 a.m. to 8:00 p.m.); ambient temperature and relative humidity were maintained at $20-25^{\circ} \mathrm{C}$ and $50 \% \pm 10 \%$. When the rats adapted to the environment 5 days later, they were randomly divided into control group $(\mathrm{C})$, model group $(\mathrm{M})$, fluoxetine group $(\mathrm{F})$, and acupuncture group (A), 12 rats in each group. All protocols were approved by the Animal Ethics Committee, Beijing University of Chinese Medicine (permission number Kj-dw-18-20140816).

2.2. The Procedures of CRS. In this study, rats in $\mathrm{C}$ were herdmanaged with 4 rats per cage, and fasting for water and food was performed from 9 a.m. to 3 p.m. every day. Each rat in $M$, $\mathrm{F}$, and A was socially isolated by placing it in a separate cage and suffered improved CRS stimulation [18, 21, 22], which restrained each rat in a self-made cylinder-shaped wire net $(20.5 \mathrm{~cm}$ long and $6.5 \mathrm{~cm}$ in diameter, fixing both ends with a butterfly clip) from 9 a.m. to 3 p.m. every day, lasting 28 days.

2.3. Fluoxetine/Acupuncture Intervention. Each rat in $\mathrm{F}$ was given fluoxetine $(10 \mathrm{mg} / \mathrm{kg})$ by gavage at one hour before the CRS procedures, once a day for 28 consecutive days. Each rat in A was acupunctured on Baihui (GV20, located at midline of the head) and Yintang (GV29, located at the midpoint between the two eyes) at one hour before the CRS procedures, once a day for 28 consecutive days $[18,23]$. Two sterilized disposable stainless steel needles $(0.18 \mathrm{~mm} \times 13 \mathrm{~mm}$, Huatuo Brand, manufactured by Suzhou Medicine Co., Ltd., Suzhou, China) were inserted obliquely into each acupoint as deep as $5 \mathrm{~mm}$ for $20 \mathrm{~min}$, once a day for 28 consecutive days.

2.4. Behavior Assays for Major Depressive Disorder. Whether the CRS treated rat in 28 days fell into depression-like behaviors was tested on days $0,7,14,21$, and 28 of the study. The open-field test was used to measure it. Four odorless plastic apparatuses with the same specifications, composed of a square arena $\left(80 \times 80 \mathrm{~cm}^{2}\right)$ with a $40 \mathrm{~cm}$ high walls, were divided into 25 equal squares $\left(16 \times 16 \mathrm{~cm}^{2}\right)$ drawn on the floor. During a $3 \mathrm{~min}$ observation period, the rat was gently placed at the center of the apparatus, and the crossing times (defined as at least three paws in a square) and the rearing times (defined as the rat standing upright on its hind legs) were recorded; then total scores of crossing and rearing were calculated.

2.5. The Frontal Cortex Dissection and Collection. Each rat was anesthetized with pentobarbital sodium (i.p., $60 \mathrm{mg} / \mathrm{kg}$ ) and rapidly euthanized. Then the frontal cortex of the rat was dissected and separated depending on "The Rat Brain Stereotaxic Coordinates" [24]. The collected tissues were frozen in liquid nitrogen and stored at $-80^{\circ} \mathrm{C}$ until processing.

2.6. RNA Extraction and RNA-Seq Experiment. Total RNA was treated with RQ1 DNase (Promega) to remove DNA. The quality and quantity of the purified RNA were determined by measuring the absorbance at $260 \mathrm{~nm} / 280 \mathrm{~nm}$ (A260/A280) using SmartSpec Plus (BioRad). RNA integrity was further verified by $1.5 \%$ agarose gel electrophoresis.

For every sample, $10 \mu \mathrm{g}$ of total RNA was used for RNAseq library preparation. Polyadenylated mRNAs were purified and concentrated with oligo (dT)-conjugated magnetic beads (invitrogen) before being used for directional RNA-seq library preparation. Purified mRNAs were iron-fragmented at $95^{\circ} \mathrm{C}$ followed by end repair and $5^{\prime}$ adaptor ligation. Then reverse transcription was performed with $\mathrm{RT}$ primer harboring $3^{\prime}$ adaptor sequence and randomized hexamer. The cDNAs were purified and amplified and PCR products corresponding to $200-500$ bps were purified, quantified, and stored at $-80^{\circ} \mathrm{C}$ until being utilised for sequencing.

For high-throughput sequencing, the libraries were prepared by following the manufacturer's instructions and applied to Illumina NextSeq 500 system with $151 \mathrm{nt}$ pair-end reads (ABLife Inc., Wuhan, China).

2.7. RNA-Seq Raw Data Clean. Raw reads were first discarded if containing more than $2-\mathrm{N}$ bases, and then reads were processed by clipping adaptor and removing low quality bases. Too short reads (less than $16 \mathrm{nt}$ ) were also dropped. FASTXToolkit (version 0.0.13) was used to get the clean reads. Quality control checks were performed by FastQC to evaluate base quality [25], GC content, sequence length distribution, and duplication level. After that, clean reads were aligned to reference genome by TopHat2 [26]. The Rattus norvegicus genome Rnor 5.0 downloaded from Ensembl was used as the reference genome. Based on gene annotation of the genome, multiple mapped reads were discarded due to their ambiguous location. Uniquely localized reads were used to calculate reads number and RPKM (reads per kilobase of exon model per million mapped reads) value of each gene [27].

The data discussed in this publication have been deposited in NCBI's Gene Expression Omnibus (GEO, https:// www.ncbi.nlm.nih.gov/geo/) under the accession number GSE86392.

2.8. Statistical Analyses. Initial processing raw data of RNA expression profiles were conducted by using edgeR software algorithm. The data of the behavior test were analyzed using SPSS 22.0 statistical software and expressed as mean \pm SEM. For each group, data at each time point were analyzed 
TABLE 1: Comparison of total scores of crossing and rearing by time $(\bar{x} \pm s, n=12)$.

\begin{tabular}{lccccc}
\hline Groups & Day 0 & Day 7 & Day 14 & Day 21 & Day 28 \\
\hline C & $75.33 \pm 8.61$ & $69.83 \pm 9.50$ & $71.83 \pm 11.22$ & $70.83 \pm 8.69$ & $66.75 \pm 7.61$ \\
M & $80.67 \pm 11.11$ & $25.75 \pm 7.12^{\star \star}$ & $16.25 \pm 5.36^{\star \star}$ & $15.08 \pm 4.36^{\star \star}$ & $15.25 \pm 3.77^{\star \star}$ \\
F & $75.67 \pm 13.90$ & $27.33 \pm 9.12$ & $32.92 \pm 16.16^{\mathbf{}}$ & $34.08 \pm 12.38^{\mathbf{\Lambda}}$ & $70.50 \pm 8.17^{\mathbf{\Delta}}$ \\
A & $73.42 \pm 12.54$ & $28.42 \pm 8.93$ & $31.08 \pm 11.21^{\mathbf{\Delta}}$ & $53.92 \pm 12.09^{\mathbf{\Delta}}$ & $72.00 \pm 8.75^{\mathbf{\Delta}}$ \\
\hline
\end{tabular}

Notes. ${ }^{\star \star} p<0.01$, versus C. ${ }^{\boldsymbol{\Lambda}} p<0.01$, versus M. Data are presented as mean \pm SEM by two-way ANOVA.

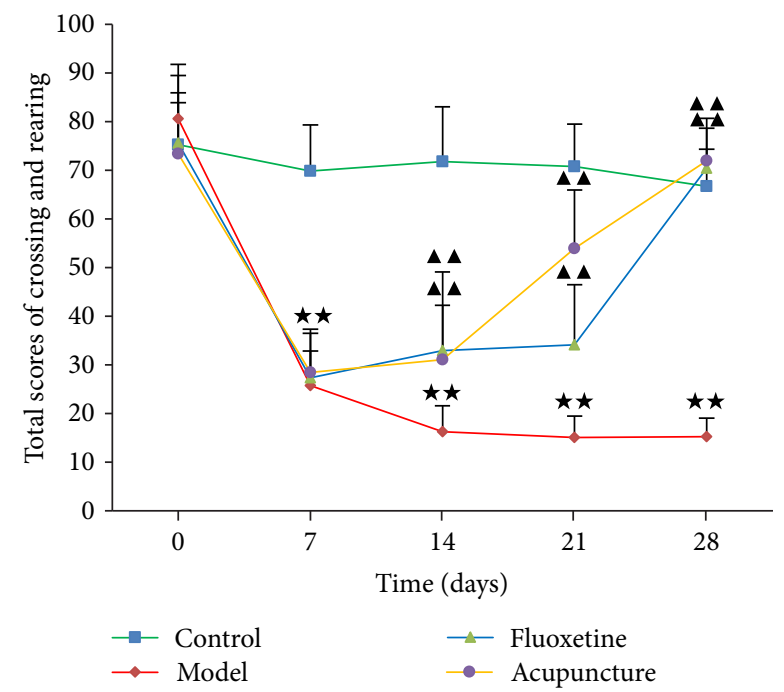

FIgURE 1: Total scores of crossing and rearing for all rats. Notes. ${ }^{\star \star} p<0.01$ versus C. ${ }^{\boldsymbol{\Delta} \Delta} p<0.01$ versus M. Data are presented as mean \pm SEM by two-way ANOVA.

applying two-way analysis of variance (ANOVA) followed by a post-hoc Tukey's test. The differences of each test are divided into the main effect, the factors, and their interaction. The difference was statistically significant $(P<0.05)$.

\section{Results}

3.1. CRS Induces the Rat to Express Depression-Like Behavior. The two-way ANOVA within days revealed total scores of crossing and rearing: the main effect $[F=71.446, p=0.000]$, effect of group $[F=163.800, p=0.000]$, effect of time $[F=127.161, p=0.000]$, and interaction between group and time $[F=29.785, p=0.000]$. As shown in Figure 1 and Table 1, before the experiment, there was no significant group difference in total scores of crossing and rearing $(p>$ 0.05). Compared to $C$, total scores of crossing and rearing of rats in $\mathrm{M}$ were significantly reduced in days $7,14,21$, and 28 ( $p<0.01, p<0.01, p<0.01$, and $p<0.01$, resp.). Compared with $\mathrm{M}$, total scores of crossing and rearing in $\mathrm{F}$ and A were significantly increased in days 14,21 , and 28 $(p<0.01, p<0.01$, and $p<0.01$, resp.).

3.2. The RNA-Seq Results. We analyzed the expression levels of frontal cortex transcriptomes, including C, M, F, and A. Based on the RPKM count data of each sample, we selected those genes that achieved a $p$ value of $<0.01$ in differential expression analysis and submitted those genes to DAVID (Database for Annotation, Visualization, and Integrated Discovery) Functional Annotation Tool (http://david.abcc .ncifcrf.gov/summary.jsp) to annotate and enrich the genes at the levels of GO and KEGG [28].

3.2.1. Differentially Expressed Genes (DEG) Analysis. We compared the expression of genes from $\mathrm{C}, \mathrm{F}$, and A with the expression of genes in $\mathrm{M}$ and analyzed the differentially expressed genes by using edgeR [29]. For each gene, significant $p$ value was obtained based on the model of negative binomial distribution. Fold changes (FC) of gene expression were also estimated in the edgeR. Genes that satisfy the following two conditions are considered as differentially expressed genes: (1) the $p$ value $<0.01$; (2) the FC level $\geq 2$ or $\leq 0.05$. Based on this definition, we identified 134,46 , and 89 differentially expressed (DE) genes for the respective C versus $M, F$ versus $M$, and A versus $M$ comparisons (Tables S1 and S2 in Supplementary Material available online at https://doi.org/10.1155/2017/1676808). In addition, DE genes in each pairwise comparison were divided into two parts: upregulated and downregulated genes. The pairwise comparison between $\mathrm{C}$ and $\mathrm{M}$ led to 20 upregulated and 114 downregulated DE genes, the pairwise comparison between $\mathrm{F}$ and $\mathrm{M}$ led to 13 upregulated and 33 downregulated $\mathrm{DE}$ genes, and the pairwise comparison between $\mathrm{A}$ and $\mathrm{M}$ led to 17 upregulated and 72 downregulated DE genes (Figure 2). In order to visualize the gene expression profiles for each group, we used Java Tree View to generate a list of genes based on thermography. As expression moves from higher to lower values, the color changes from red to green (Figure 3). In order to examine the overlap among the DE genes of each pairwise comparison, we used a Venn diagram to display the results (Figure 4). There were 8 common DE genes among $C$ versus $M, F$ versus $M$, and $A$ versus $M$, including 1 upregulated gene and 7 downregulated genes; 6 common DE genes between $C$ versus $M$ and $F$ versus $M$, including 2 upregulated and 4 downregulated genes; and 18 common $\mathrm{DE}$ genes between $\mathrm{C}$ versus $\mathrm{M}$ and $\mathrm{A}$ versus $\mathrm{M}$, including 3 upregulated and 15 downregulated genes (Table 2).

3.2.2. GO Term Enrichment Analysis. GO is an international standardized gene function classification system, which used to describe the characteristics of genes and their products in any organism. Using the GO term enrichment analysis, one or a group of genes can be classified according to the three aspects of biological process (BP), molecular function 


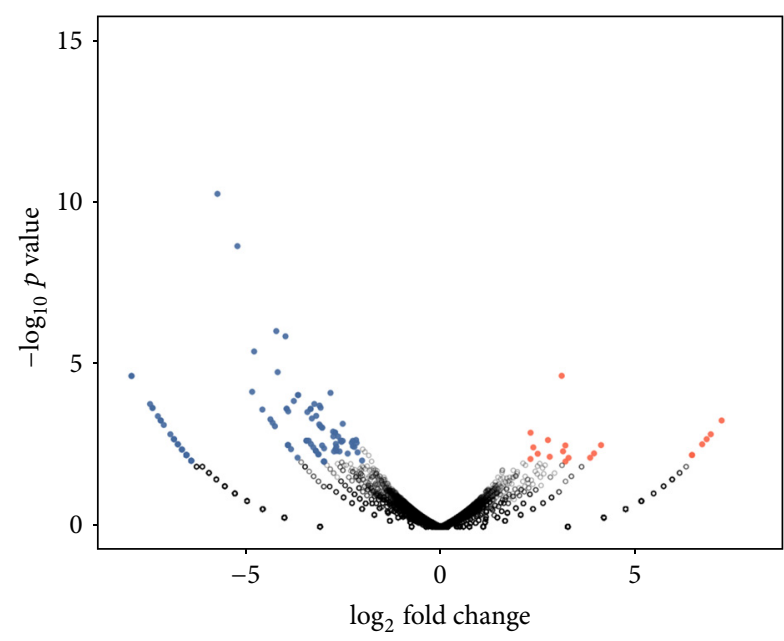

- Not differentially expressed (20725)

- Downregulated genes (114)

- Upregulated genes (20)

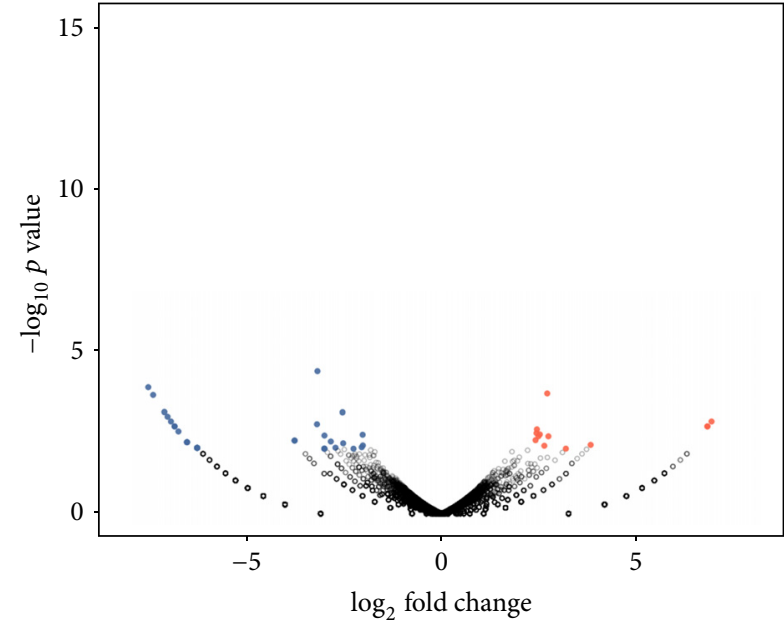

- Not differentially expressed (20813)

- Downregulated genes (33)

- Upregulated genes (13)

(a)

(b)

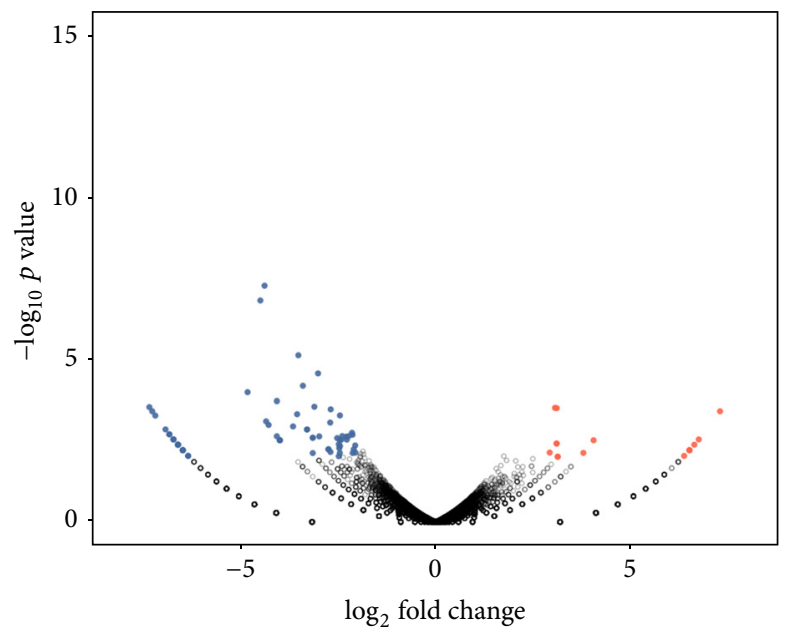

- Not differentially expressed (20770)
- Downregulated genes (72)
- Upregulated genes (17)

(c)

FIGURE 2: Volcano plots from pairwise comparisons of the samples. (a) Volcano plots of genes from the pairwise comparison of C versus M. (b) Volcano plots of genes from the pairwise comparison of F versus M. (c) Volcano plots of genes from the pairwise comparison of A versus M.

(MF), and cellular component (CC). Therefore, in order to further investigate differences between the DE genes, GO term enrichment analysis was applied to search the enriched biological function items. In this analysis, GO terms with corrected $p$ value $<0.05$ were considered as significantly enriched. We tested the enrichment of terms for each gene list and only identified the significantly enriched GO terms in the downregulated $\mathrm{DE}$ genes for the $C$ versus $\mathrm{M}, \mathrm{F}$ versus $\mathrm{M}$, and $A$ versus $M$ comparisons, followed by 32, 1, and 15 (Table S3, Figure 5). The Venn diagram to illustrate the overlap of the significantly enriched GO terms was shown in Figure 6. There was 1 GO term commonly significantly enriched in the downregulated DE genes among the three comparisons, which were of extracellular space. Notably, there were 8 GO terms commonly significantly enriched in the downregulated DE genes between the comparisons of $C$ versus $M$ and A versus $M$, mainly involved in the cellular response to lipopolysaccharide, inflammatory response, molecular function, and external side of plasma membrane (Table 3 ).

3.2.3. KEGG Pathway Enrichment Analysis. In an organism, the gene product does not exist in isolation, and the specific biological functions of different gene products are coordinated through the orderly coordination. KEGG is a collection 


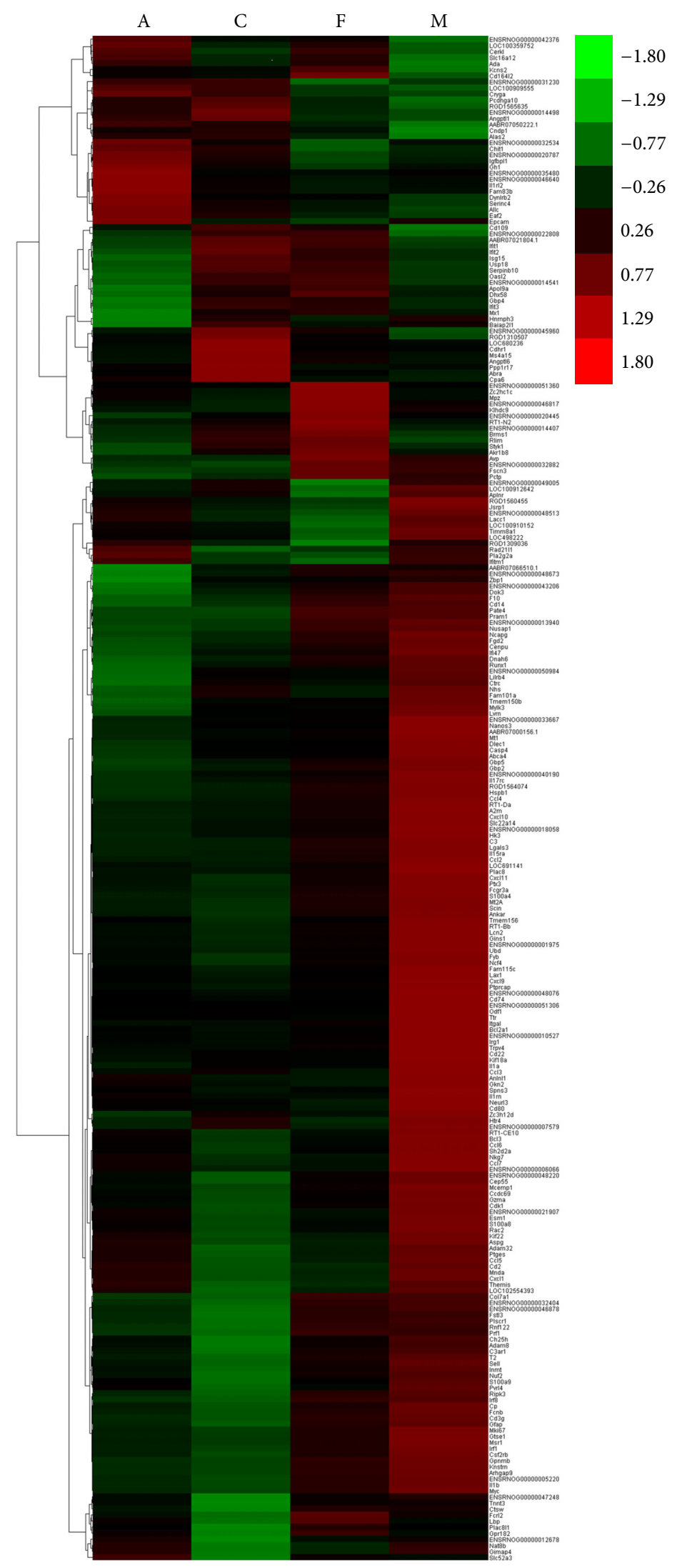

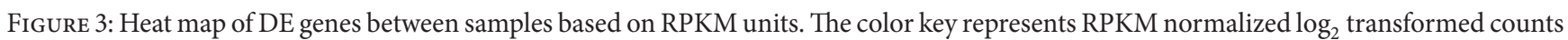
and each row represents a gene. 


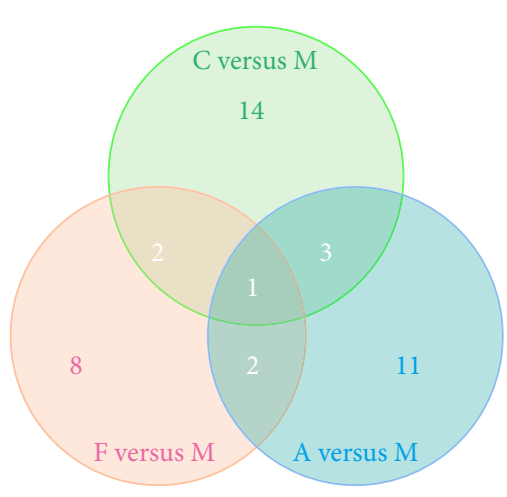

(a)

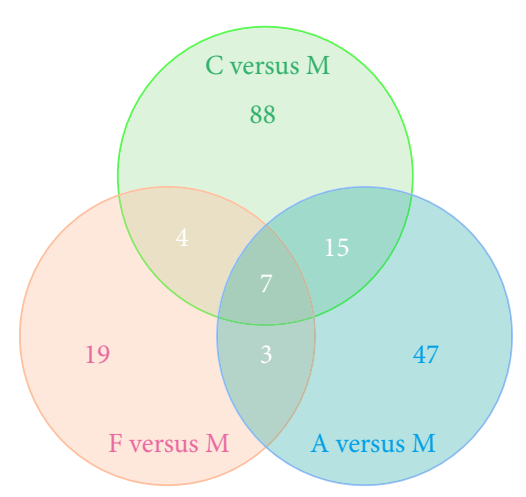

(b)

FIGURE 4: Venn diagrams showing the overlap of DE genes from pairwise comparisons of the samples. (a) Out of 41 upregulated DE genes identified as differentially expressed in at least two pairwise comparisons of the samples, 1 upregulated gene was shared across all sample pairwise comparisons. (b) Out of 183 downregulated DE genes identified as differentially expressed in at least two pairwise comparisons of the samples, 7 downregulated genes were shared across all sample pairwise comparisons.

TABLE 2: Common significant DE genes.

\begin{tabular}{|c|c|c|}
\hline \multirow{2}{*}{ Samples } & \multicolumn{2}{|c|}{ DE genes } \\
\hline & Upregulated & Downregulated \\
\hline $\begin{array}{l}C \text { versus } M \cap F \text { versus } M \cap A \\
\text { versus } M\end{array}$ & Pcdhgb2 & $\begin{array}{c}\text { Ubd, Ttr, Cxcl9, Serpina3n, } \\
\text { Irg1, Odf1, Trpv4 }\end{array}$ \\
\hline F versus $M \cap C$ versus $M$ & Ifit1, LOC100912860 & $\begin{array}{c}\text { AABR06089930.1, Cd80, } \\
\text { Tfec, Illrn }\end{array}$ \\
\hline A versus $M \cap C$ versus $M$ & Alas2, Angptl1, Cndp1 & $\begin{array}{c}\text { Plac8, C3, Bcl2a1, S100a4, } \\
\text { Il15ra, Ptprcap, Lgals3, } \\
\text { Ccl2, LOC691141, Ptx3, } \\
\text { Itgal, Pram1, Hk3, Pate4, } \\
\text { A2m }\end{array}$ \\
\hline F versus $M \cap A$ versus $M$ & Ada, Slc16a12 & Hsf5, Rn50_1_2682.1, Cd22 \\
\hline
\end{tabular}

TABLE 3: Common significant GO terms for DE genes.

\begin{tabular}{|c|c|c|}
\hline \multirow{2}{*}{ Samples } & \multicolumn{2}{|c|}{ GO terms } \\
\hline & Upregulated & Downregulated \\
\hline $\begin{array}{l}C \text { versus } M \cap F \text { versus } M \cap A \\
\text { versus } M\end{array}$ & - & CC: extracellular space \\
\hline $\mathrm{F}$ versus $\mathrm{M} \cap \mathrm{C}$ versus $\mathrm{M}$ & - & - \\
\hline$A$ versus $M \cap C$ versus $M$ & - & $\begin{array}{l}\text { BP: cellular response to } \\
\text { lipopolysaccharide } \\
\text { BP: response to } \\
\text { lipopolysaccharide } \\
\text { BP: inflammatory response } \\
\text { MF: molecular function } \\
\text { CC: external side of plasma } \\
\text { membrane } \\
\text { MF: protein } \\
\text { heterodimerization activity } \\
\text { CC: extracellular vesicular } \\
\text { exosome } \\
\text { CC: cellular component }\end{array}$ \\
\hline$F$ versus $M \cap A$ versus $M$ & - & - \\
\hline
\end{tabular}




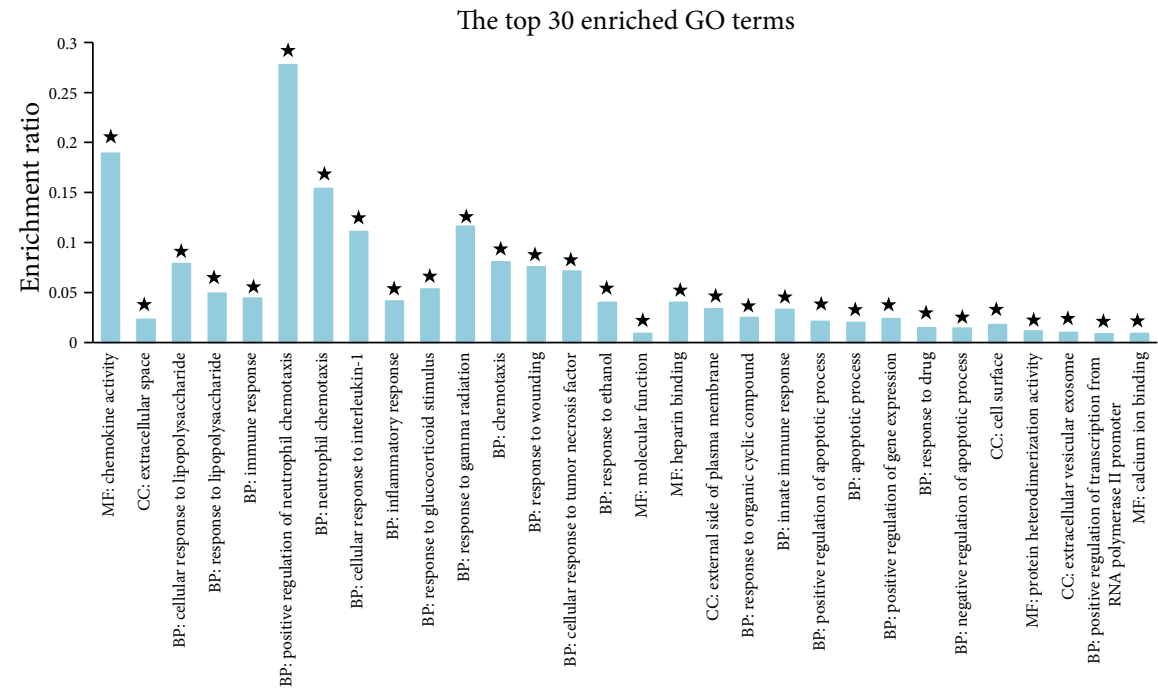

(a)

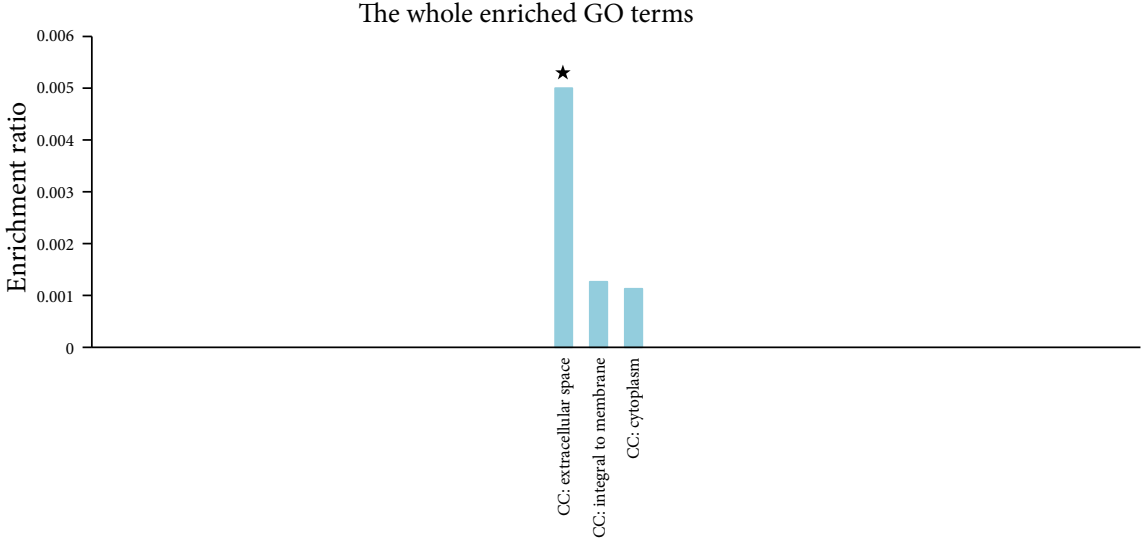

(b)

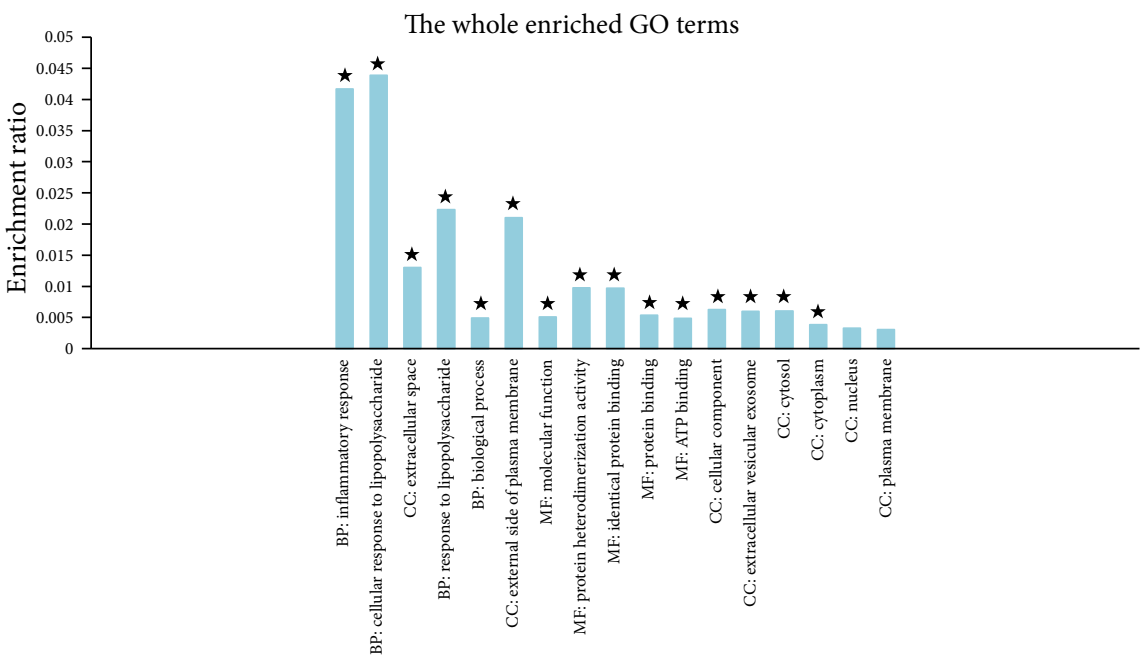

(c)

FIGURE 5: GO term enrichment analysis of DE genes from pairwise comparisons between the samples. (a) Histogram of the enriched GO terms of downregulated $\mathrm{DE}$ genes from the pairwise comparison of $\mathrm{C}$ versus $\mathrm{M}$. The significance of each $\mathrm{GO}$ term was estimated based on corrected $p$ values (corrected $p<0.05$ ), which was labeled with $\star$ on each histogram. (b) Histogram of the enriched GO terms of downregulated DE genes from the pairwise comparison of F versus M. The significance of each GO term was estimated based on corrected $p$ values (corrected $p<0.05$ ), which was labeled with $\star$ on each histogram. (c) Histogram of the enriched GO terms of downregulated DE genes from the pairwise comparison of A versus M. The significance of each GO term was estimated based on corrected $p$ values (corrected $p$ value $<0.05$ ), which was labeled with $\star$ on each histogram. 
TABLE 4: Common significant KEGG pathways for DE genes.

\begin{tabular}{|c|c|c|}
\hline \multirow{2}{*}{ Samples } & \multicolumn{2}{|c|}{ KEGG pathways } \\
\hline & Upregulated & Downregulated \\
\hline $\begin{array}{l}C \text { versus } M \cap F \text { versus } M \cap A \\
\text { versus } M\end{array}$ & - & $\begin{array}{l}\text { OS: Toll-like receptor signaling } \\
\text { pathway }\end{array}$ \\
\hline $\mathrm{F}$ versus $\mathrm{M} \cap \mathrm{C}$ versus $\mathrm{M}$ & - & - \\
\hline A versus $M \cap C$ versus $M$ & $\begin{array}{l}\text { M: histidine metabolism } \\
\text { M: beta-alanine } \\
\text { metabolism } \\
\text { M: glycine, serine, and } \\
\text { threonine metabolism } \\
\text { M: porphyrin and } \\
\text { chlorophyll } \\
\text { metabolism }\end{array}$ & $\begin{array}{c}\text { OS: chemokine signaling } \\
\text { pathway } \\
\text { EIP: cytokine-cytokine } \\
\text { receptor interaction } \\
\text { EIP: TNF signaling } \\
\text { pathway } \\
\text { HD: rheumatoid arthritis } \\
\text { HD: Staphylococcus aureus } \\
\text { infection } \\
\text { HD: pertussis } \\
\text { HD: malaria } \\
\text { OS: cytosolic } \\
\text { DNA-sensing pathway } \\
\text { HD: legionellosis }\end{array}$ \\
\hline F versus $M \cap A$ versus $M$ & - & - \\
\hline
\end{tabular}

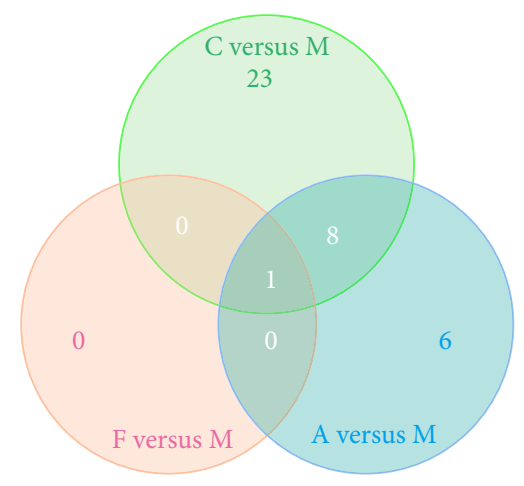

FIGURE 6: Venn diagrams showing the overlap of significant GO terms for downregulated $\mathrm{DE}$ genes from pairwise comparisons of the samples. Out of 38 significant GO terms of downregulated DE genes identified as differentially expressed in at least two pairwise comparisons of the samples, 1 significant GO term was shared across all sample pairwise comparisons.

of genomic and metabolic pathways, as well as database integration with new biochemical pathways. KEGG analysis is on the pathway enrichment analysis when considering the pathways topology structure and the position of each gene in the channel. The output includes pathways interest rates and the corresponding significance level ( $p$ value) [30, 31]. In this analysis, KEGG pathways with corrected $p$ value $<0.05$ were regarded as significantly enriched. For the upregulated DE genes, we finally identified 4,0 , and 8 significantly enriched KEGG pathways for the $\mathrm{C}$ versus $\mathrm{M}$, $\mathrm{F}$ versus $\mathrm{M}$, and $\mathrm{A}$ versus M comparisons, respectively (Table S4, Figure 7). The Venn diagram to illustrate the overlap of the significantly enriched KEGG pathways was shown in Figure 8(a); there were 4
KEGG pathways commonly significantly enriched in the upregulated DE genes between the comparisons of A versus $\mathrm{M}$ and $\mathrm{C}$ versus $\mathrm{M}$, which were of histidine metabolism, beta-alanine metabolism, and glycine, serine, and threonine metabolism. In the downregulated DE genes, we identified 17, 1 , and 16 significantly enriched KEGG pathways, respectively, for the $C$ versus $M$, $F$ versus $M$, and $A$ versus $M$ comparisons (Table S5, Figure 9). The Venn diagram to illustrate the overlap of the significantly enriched KEGG pathways was shown in Figure 8(b). There was 1 KEGG pathway commonly significantly enriched in the downregulated DE genes among the three comparisons, which was the Toll-like receptor signaling pathway. Moreover, there were 9 KEGG pathways commonly significantly enriched in the downregulated $\mathrm{DE}$ genes between $C$ versus $M$ and $A$ versus $M$ comparisons and which were mainly involved in chemokine signaling pathway, TNF signaling pathway, NF-kappa B signaling pathway, and cytosolic DNA-sensing pathway (Table 4).

\section{Discussion}

In this study, we used open-field test to ascertain whether acupuncture affects CRS induced depression-like behaviors in rats, and explored the antidepressant mechanism of acupuncture at the molecular level of transcriptome in the frontal cortex of CRS rats. Here, we identified some interesting findings.

Based on the results of open-field test, the ability to move and explore decreased in $\mathrm{M}$, which showed that CRS successfully induced depression-like behaviors in rats. In F and A, both interventions significantly reversed these behavioral changes, indicating antidepressant effects on CRS rats.

DEG analysis showed that there were obvious differences among the three comparisons of $C$ versus $M, F$ versus $M$, 


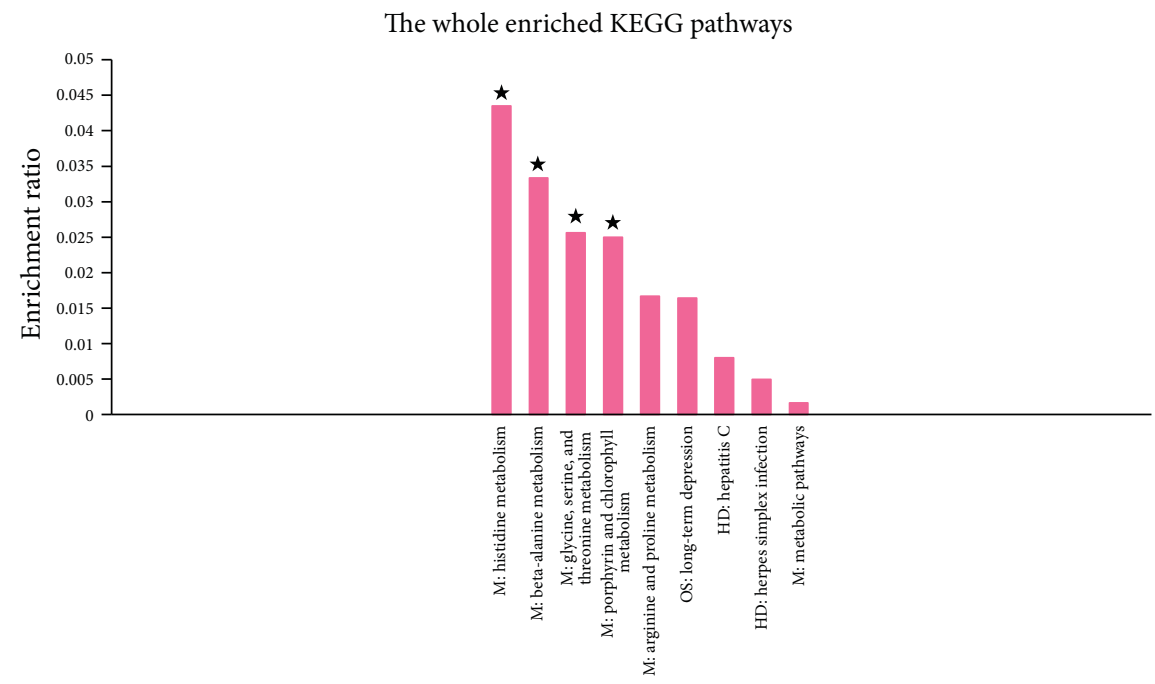

(a)

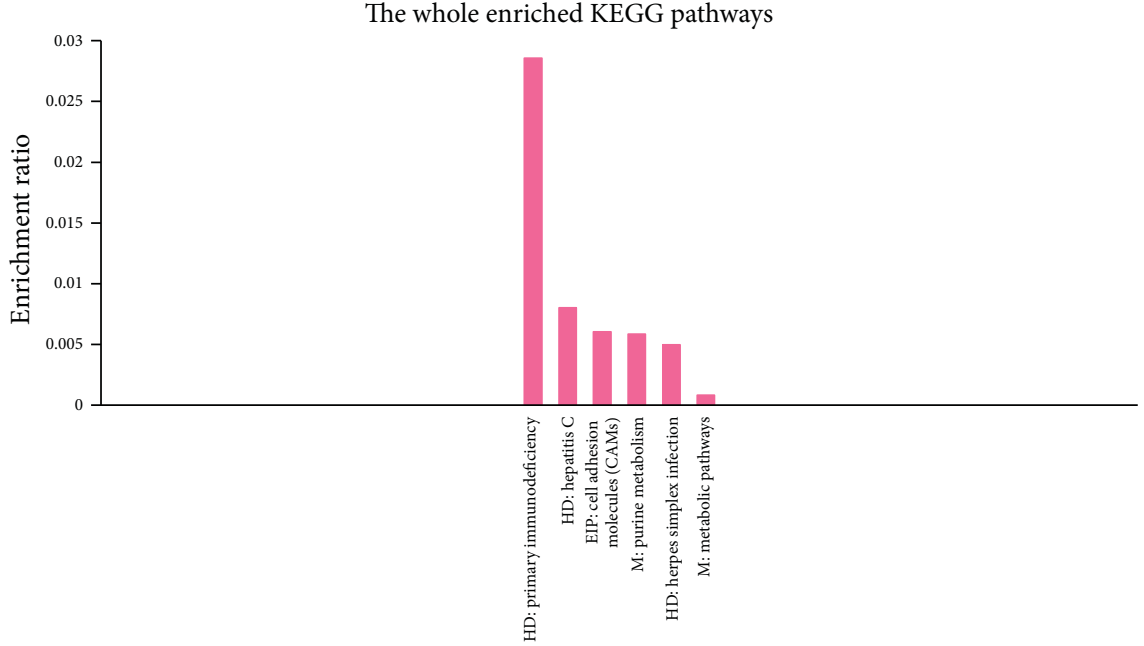

(b)

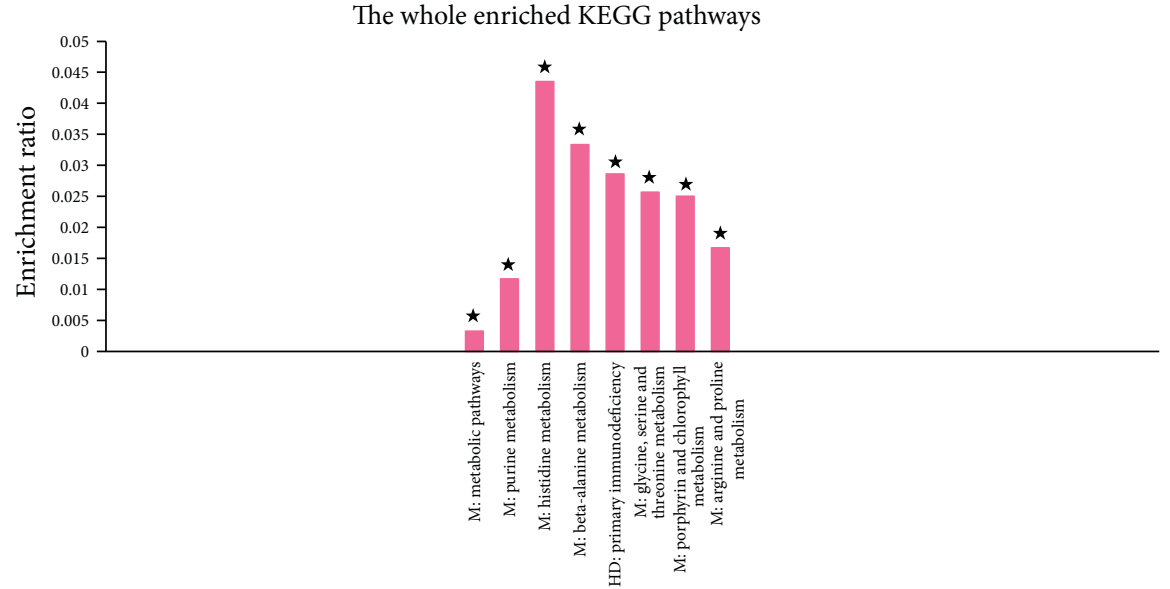

(c)

FIGURE 7: KEGG pathway enrichment analysis of upregulated DE genes from pairwise comparisons between the samples. (a) Histogram of the enriched KEGG pathways of upregulated DE genes from the pairwise comparison of C versus M. The significance of each KEGG pathway was estimated based on corrected $p$ values (corrected $p<0.05$ ), which was labeled with $\star$ on each histogram. (b) Histogram of the enriched KEGG pathways of upregulated DE genes from the pairwise comparison of F versus $\mathrm{M}$. The significance of each KEGG pathway was estimated based on corrected $p$ values (corrected $p<0.05$ ), which was labeled with $\star$ on each histogram. (c) Histogram of the enriched KEGG pathways of upregulated DE genes from the pairwise comparison of A versus M. The significance of each KEGG pathway was estimated based on corrected $p$ values (corrected $p<0.05$ ), which was labeled with $\star$ on each histogram. 


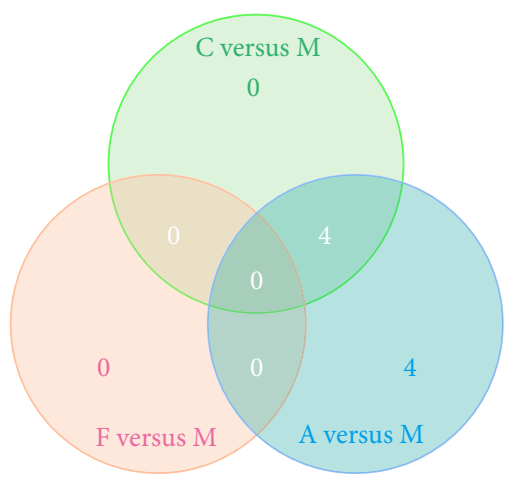

(a)

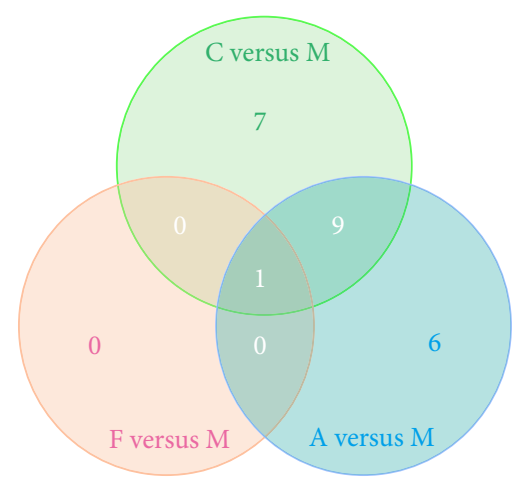

(b)

FIGURE 8: Venn diagrams showing the overlap of significant KEGG pathways of DE genes from the pairwise comparisons of the samples. (a) Out of 8 significant KEGG pathways of upregulated DE genes identified as differentially expressed in at least two pairwise comparisons of the samples, there were no significant KEGG pathways shared across all sample pairwise comparisons. (b) Out of 23 significant KEGG pathways of downregulated DE genes identified as differentially expressed in at least two pairwise comparisons of the samples, there was 1 significant KEGG pathway shared across all sample pairwise comparisons.

and $\mathrm{A}$ versus $\mathrm{M}$, followed by 134,46 , and $89 \mathrm{DE}$ genes. In total of 269 DE genes among the three comparisons, only 8 DE genes overlapped, including Pcdhgb2, Ubd, Ttr, Cxcl9, Serpina3n, Irg1, Odf1, and Trpv4, but there are few reports of depression associated with these genes, except the $\operatorname{Ttr}[32,33]$. Notably, there were 18 common genes differentially expressed between $C$ versus $M$ and $A$ versus $M$, and these genes are mainly involved in neurodevelopment, inflammation, signal transduction, and cellular communication; in addition, genes including Alas2 [34], C3 [35], Ccl2 [36-38], and Ptx3 [39] have been reported to be associated with depression; the others were rarely reported. These results suggest that acupuncture intervention may exert an antidepressant effect by influencing the expression of these genes in the frontal cortex.

To further clarify these differences among the three comparisons, we used GO term enrichment analysis and KEGG pathway enrichment analysis for these DE genes. In the GO enrichment analysis, there were $8 \mathrm{GO}$ terms commonly significantly enriched in the downregulated DE genes between the comparisons of $C$ versus $M$ and $A$ versus $\mathrm{M}$, mainly involved in the cellular response to lipopolysaccharide, inflammatory response, molecular function, and external side of plasma membrane. In KEGG pathway enrichment analysis, there were 4 KEGG pathways commonly significantly enriched in the upregulated DE genes between the comparisons of $A$ versus $M$ and $C$ versus $M$, which were of histidine metabolism, beta-alanine metabolism, and glycine, serine and threonine metabolism, and $10 \mathrm{KEGG}$ pathways commonly significantly enriched in the downregulated DE genes between $C$ versus $M$ and $A$ versus $M$ comparisons and which were mainly involved in Toll-like receptor signaling pathway, chemokine signaling pathway, TNF signaling pathway, NF-kappa B signaling pathway, and cytosolic DNA-sensing pathway. These findings not only support the view that depression is associated with neuroendocrine and inflammatory pathways, but also indicate that the antidepressant effects of acupuncture may play a role in regulating amino acid metabolism and inflammatory related pathways in the frontal cortex, especially the Tolllike receptor signaling pathway, TNF signaling pathway, and NF-kappa B signaling pathway. Interestingly, the Tolllike receptor signaling pathway and NF-kappa B signaling pathway are closely related which is based on the pathway diagram provided by KEGG database; and our previous study also suggested that the antidepressant effect of acupuncture might be mediated by inhibition of inflammatory mediators via modulation of NF-kappa B in the frontal cortex [20].

\section{Conclusion}

In this study, some of the key molecular signatures coincided with important pathological features observed in previous reports; however, many molecules have not been reported in clinical and experimental studies of depression, which suggests the pathogenesis of depression is sophisticated. Further, it is precisely the pathological complexity of depression that has led to inefficiencies in the development of antidepressant drugs over the past few decades [40]. But what is astonishing is that the number of common DE genes/GO terms/KEGG pathways between the $C$ versus $M$ and $A$ versus $M$ comparisons is more than the comparisons of $C$ versus $M$ and $F$ versus $M$, combined with the behavioral findings; it suggests the antidepressant effect of acupuncture may be achieved by targeting multiple targets. To some extent, this is in line with the view that it may be more effective to target psychosis with multiple target drugs rather than single target drugs [40]. More importantly, the multitarget antidepressant effect may be related to amino acid metabolism and inflammatory pathways, the Toll-like receptor signaling pathway and the NF-kappa B signaling pathway may be the two important pathways for acupuncture antidepressant.

However, what needs to be emphasized here is that all of the inferences above-mentioned are based on the results of this study, and the conjecture has not been verified. So, based 


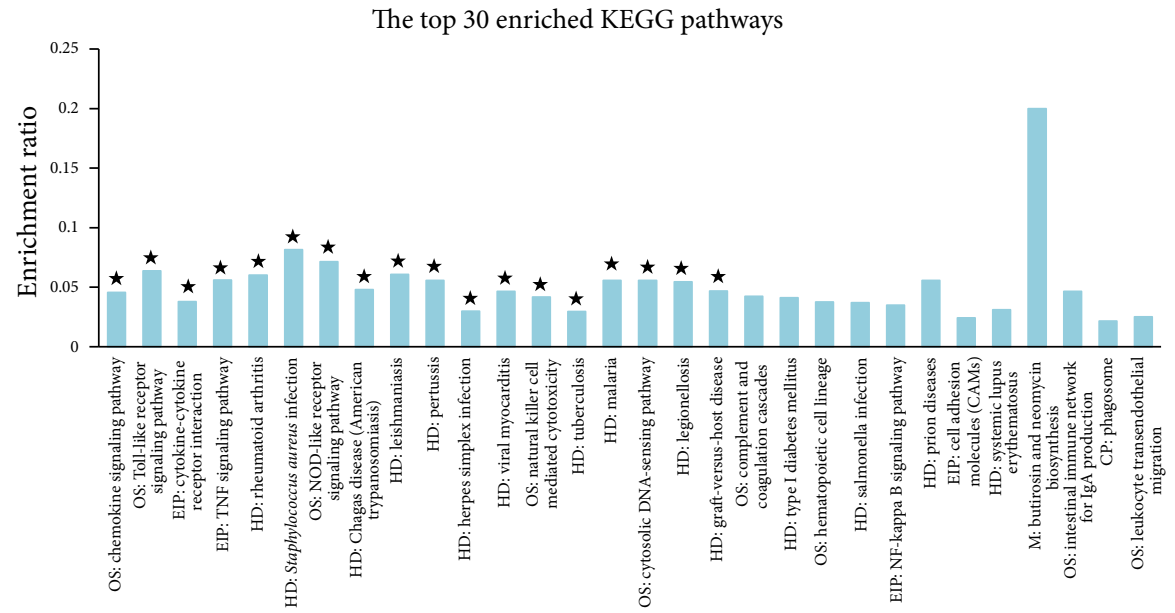

(a)

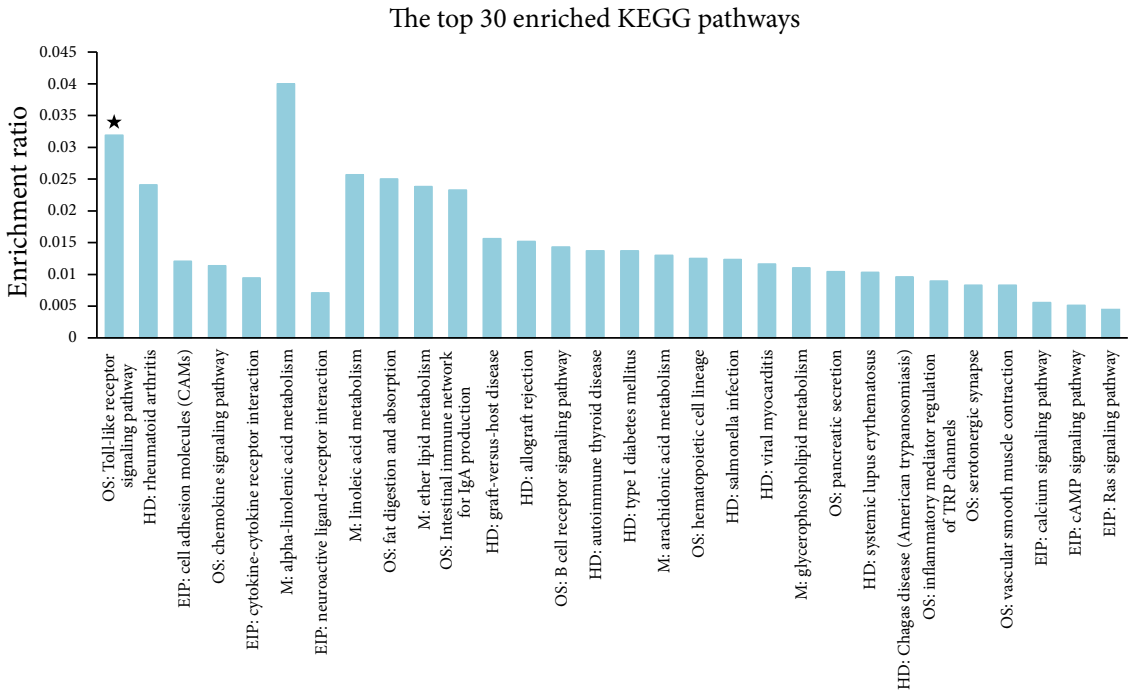

(b)

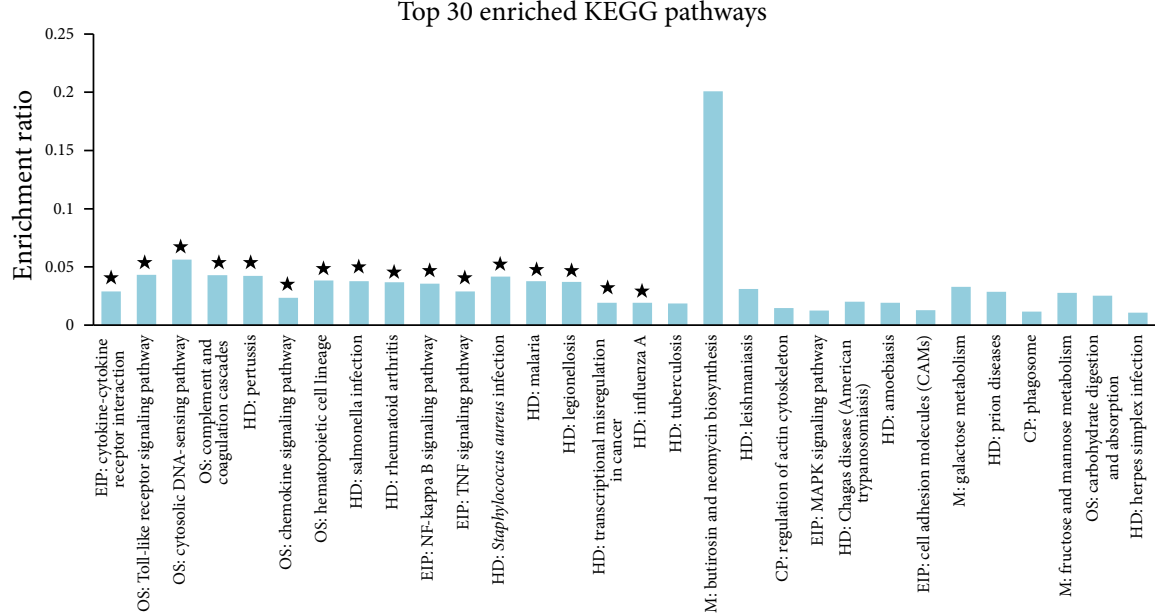

(c)

FIGURE 9: KEGG pathway enrichment analysis of downregulated DE genes from pairwise comparisons between the samples. (a) Histogram of the enriched KEGG pathways of downregulated DE genes from the pairwise comparison of $\mathrm{C}$ versus M. The significance of each KEGG pathway was estimated based on corrected $p$ values (corrected $p<0.05$ ), which was labeled with $\star$ on each histogram. (b) Histogram of the enriched KEGG pathways of downregulated DE genes from the pairwise comparison of F versus M. The significance of each KEGG pathway was estimated based on corrected $p$ values (corrected $p<0.05$ ), which was labeled with $\star$ on each histogram. (c) Histogram of the enriched KEGG pathways of downregulated DE genes from the pairwise comparison of A versus $M$. The significance of each KEGG pathway was estimated based on corrected $p$ values (corrected $p<0.05$ ), which was labeled with $\star$ on each histogram. 
on the scientific objectivity, we will provide more experimental evidence for antidepressant effect of acupuncture through further exploration in our next study.

\section{Conflicts of Interest}

The authors declare no financial interests or other conflicts of interest.

\section{Authors' Contributions}

Yu Wang and Huili Jiang contributed equally to this work.

\section{Acknowledgments}

The authors' work reported here was supported by the Outstanding Doctoral Dissertation Guidance Teachers of Science and Technology Projects of Beijing (Grant no. YB20091002601) and the National Natural Science Foundation of China (Grant no. 81373729).

\section{References}

[1] T.-L. Huang and C.-C. Lin, "Advances in biomarkers of major depressive disorder," Advances in Clinical Chemistry, vol. 68, pp. 177-204, 2015.

[2] J. D. Bremner, M. Narayan, E. R. Anderson, L. H. Staib, H. L. Miller, and D. S. Charney, "Hippocampal volume reduction in major depression," The American Journal of Psychiatry, vol. 157, no. 1, pp. 115-118, 2000.

[3] T.-J. Lai, M. E. Payne, C. E. Byrum, D. C. Steffens, and K. R. R. Krishnan, "Reduction of orbital frontal cortex volume in geriatric depression," Biological Psychiatry, vol. 48, no. 10, pp. 971-975, 2000.

[4] W. C. Drevets, J. L. Price, and M. L. Furey, "Brain structural and functional abnormalities in mood disorders: implications for neurocircuitry models of depression," Brain Structure and Function, vol. 213, no. 1-2, pp. 93-118, 2008.

[5] K. Ma, L. Guo, A. Xu, S. Cui, J. Wang, and K. Hashimoto, "Molecular mechanism for stress-induced depression assessed by sequencing miRNA and mRNA in medial prefrontal cortex," PLoS One, vol. 11, no. 7, Article ID e0159093, 2016.

[6] V. Krishnan and E. J. Nestler, "The molecular neurobiology of depression," Nature, vol. 455, no. 7215, pp. 894-902, 2008.

[7] G. E. Hodes, V. Kana, C. Menard, M. Merad, and S. J. Russo, "Neuroimmune mechanisms of depression," Nature Neuroscience, vol. 18, no. 10, pp. 1386-1393, 2015.

[8] R. N. Schnyer and J. J. Allen, Acupuncture in the Treatment of Depression: A Manual for Practice and Research, Churchill Livingstone, London, England, 2001.

[9] H. Macpherson, B. Elliot, A. Hopton, H. Lansdown, and S. Richmond, "Acupuncture for depression: patterns of diagnosis and treatment within a randomised controlled trial," Evidencebased Complementary and Alternative Medicine, vol. 2013, Article ID 286048, 9 pages, 2013.

[10] D. P. Sniezek and I. J. Siddiqui, "Acupuncture for treating anxiety and depression in women: a clinical systematic review," Medical Acupuncture, vol. 25, no. 3, pp. 164-172, 2013.
[11] H. Zhao, Y.-X. Sun, H.-C. Luo et al., "Clinical practice guidelines for using acupuncture to treat depression," Chinese Journal of Integrative Medicine, pp. 1-10, 2015.

[12] B. Dong, Z. Chen, X. Yin et al., "The efficacy of acupuncture for treating depression-related insomnia compared with a control group: a systematic review and meta-analysis," BioMed Research International, vol. 2017, Article ID 9614810, 11 pages, 2017.

[13] F. Ling, F. Wenbin, C. Zhao et al., "Curative effect of acupuncture on quality of life in patient with depression: a clinical randomized single-blind placebo-controlled study," Journal of Traditional Chinese Medicine, vol. 36, no. 2, pp. 151-159, 2016.

[14] D. Martins-de-Souza, "Proteomics, metabolomics, and protein interactomics in the characterization of the molecular features of major depressive disorder," Dialogues in Clinical Neuroscience, vol. 16, no. 1, pp. 63-73, 2014.

[15] A. H. Miller and C. L. Raison, "The role of inflammation in depression: from evolutionary imperative to modern treatment target," Nature Reviews Immunology, vol. 16, no. 1, pp. 22-34, 2016.

[16] J. Lu, J. Liang, and J. R. Wang, "Acupuncture activates ERKCREB pathway in rats exposed to chronic unpredictable mild stress," Evidence-Based Complementary and Alternative Medicine, vol. 2013, Article ID 469765, 7 pages, 2013.

[17] T. W. Guo, Z. Guo, X. J. Yang et al., "The alterations of IL1beta, IL-6, and TGF-beta levels in hippocampal CA3 region of chronic restraint stress rats after Electroacupuncture (EA) pretreatment," Evidence-Based Complementary and Alternative Medicine, vol. 2014, Article ID 369158, 7 pages, 2014.

[18] Z. Guo, Y. Tu, T.-W. Guo et al., "Electroacupuncture pretreatment exhibits anti-depressive effects by regulating hippocampal proteomics in rats with chronic restraint stress," Neural Regeneration Research, vol. 10, no. 8, pp. 1298-1304, 2015.

[19] X. Zhang, Y. Song, T. Bao et al., "Antidepressant-like effects of acupuncture involved the ERK signaling pathway in rats," $B M C$ Complementary and Alternative Medicine, vol. 16, no. 1, article no. 380, 2016.

[20] J. Lu, R.-H. Shao, S.-Y. Jin, L. Hu, Y. Tu, and J.-Y. Guo, "Acupuncture ameliorates inflammatory response in a chronic unpredictable stress rat model of depression," Brain Research Bulletin, vol. 128, pp. 106-112, 2017.

[21] R. J. Tynan, S. Naicker, M. Hinwood et al., "Chronic stress alters the density and morphology of microglia in a subset of stressresponsive brain regions," Brain, Behavior, and Immunity, vol. 24, no. 7, pp. 1058-1068, 2010.

[22] M. Hinwood, J. Morandini, T. A. Day, and F. R. Walker, "Evidence that microglia mediate the neurobiological effects of chronic psychological stress on the medial prefrontal cortex," Cerebral Cortex, vol. 22, no. 6, pp. 1442-1454, 2012.

[23] Q. Liu, B. Li, H.-Y. Zhu, Y.-Q. Wang, J. Yu, and G.-C. Wu, "Glia atrophy in the hippocampus of chronic unpredictable stressinduced depression model rats is reversed by electroacupuncture treatment," Journal of Affective Disorders, vol. 128, no. 3, pp. 309-313, 2011.

[24] G. Paxinos and C. Watson, The Rat Brain in Stereotaxic Coordinates, Academic Press, Cambridge, England, 6th edition, 2007.

[25] S. Andrews, "FastQC: A quality control tool for high throughput sequence data," http://www.bioinformatics.babraham.ac.uk/ projects/fastqc/.

[26] D. Kim, G. Pertea, C. Trapnell, H. Pimentel, R. Kelley, and S. L. Salzberg, "TopHat2: accurate alignment of transcriptomes in the presence of insertions, deletions and gene fusions," Genome Biology, vol. 14, no. 4, article R36, 2013. 
[27] A. Mortazavi, B. A. Williams, K. McCue, L. Schaeffer, and B. Wold, "Mapping and quantifying mammalian transcriptomes by RNA-Seq," Nature Methods, vol. 5, no. 7, pp. 621-628, 2008.

[28] G. Dennis, B. T. Sherman, and D. A. Hosack, "DAVID: database for annotation, visualization, and integrated discovery," Genome Biology, vol. 4, no. 5, article 3, 2003.

[29] M. D. Robinson, D. J. McCarthy, and G. K. Smyth, "edgeR: a Bioconductor package for differential expression analysis of digital gene expression data.," Bioinformatics, vol. 26, no. 1, pp. 139-140, 2010.

[30] S. Draghici, P. Khatri, A. L. Tarca et al., "A systems biology approach for pathway level analysis," Genome Research, vol. 17, no. 10, pp. 1537-1545, 2007.

[31] J. Wang and M. D. Li, "Common and unique biological pathways associated with smoking initiation/progression, nicotine dependence, and smoking cessation," Neuropsychopharmacology, vol. 35, no. 3, pp. 702-719, 2010.

[32] G. M. Sullivan, J. J. Mann, M. A. Oquendo, E. S. Lo, T. B. Cooper, and J. M. Gorman, "Low cerebrospinal fluid transthyretin levels in depression: correlations with suicidal ideation and low serotonin function," Biological Psychiatry, vol. 60, no. 5, pp. 500506, 2006.

[33] L. Dixson, K. Ridler, T. E. Nichols et al., "Thyroid hormone transporter genes and grey matter changes in patients with major depressive disorder and healthy controls," Psychoneuroendocrinology, vol. 36, no. 6, pp. 929-934, 2011.

[34] Y. Yamamoto, T. Ueyama, T. Ito, and Y. Tsuruo, "Downregulation of growth hormone 1 gene in the cerebellum and prefrontal cortex of rats with depressive-like behavior," Physiological Genomics, vol. 47, no. 5, pp. 170-176, 2015.

[35] N. Strenn, P. Suchankova, S. Nilsson et al., "Expression of inflammatory markers in a genetic rodent model of depression," Behavioural Brain Research, vol. 281, pp. 348-357, 2015.

[36] C.-U. Pae, "Monocyte chemoattractant protein-1 (MCP1) may play a role in depression," Progress in Neuro-Psychopharmacology and Biological Psychiatry, vol. 32, no. 1, pp. 313-314, 2008.

[37] S. M. Lehto, L. Niskanen, K.-H. Herzig et al., "Serum chemokine levels in major depressive disorder," Psychoneuroendocrinology, vol. 35, no. 2, pp. 226-232, 2010.

[38] J. E. Piletz, A. Halaris, O. Iqbal et al., "Pro-inflammatory biomakers in depression: treatment with venlafaxine," World Journal of Biological Psychiatry, vol. 10, no. 4, pp. 313-323, 2009.

[39] R. C. Shelton, S. Liang, P. Liang, A. Chakrabarti, D. H. Manier, and F. Sulser, "Differential expression of pentraxin 3 in fibroblasts from patients with major depression," Neuropsychopharmacology, vol. 29, no. 1, pp. 126-132, 2004.

[40] M.-M. Poo, J.-L. Du, N. Y. Ip, Z.-Q. Xiong, B. Xu, and T. Tan, "China brain project: basic neuroscience, brain diseases, and brain-inspired computing," Neuron, vol. 92, no. 3, pp. 591-596, 2016. 


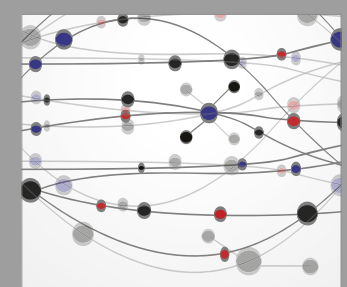

The Scientific World Journal
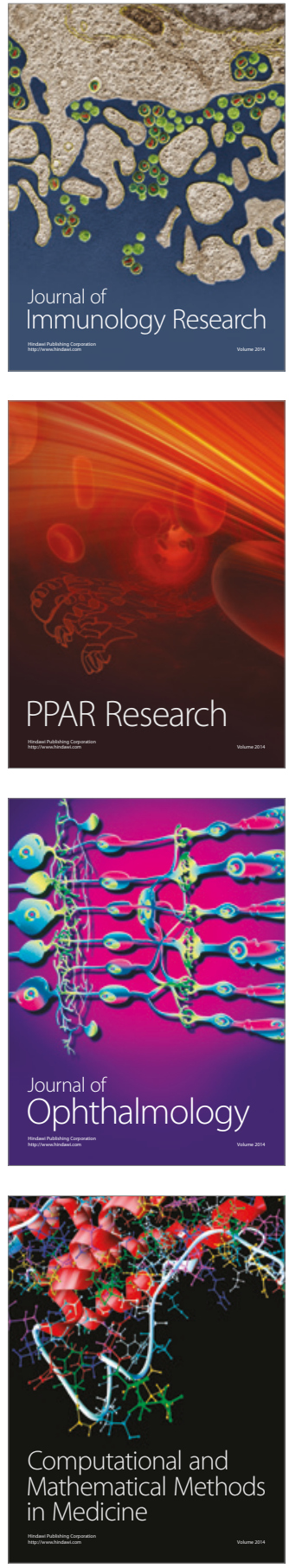

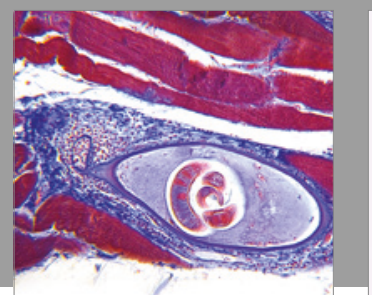

Gastroenterology Research and Practice
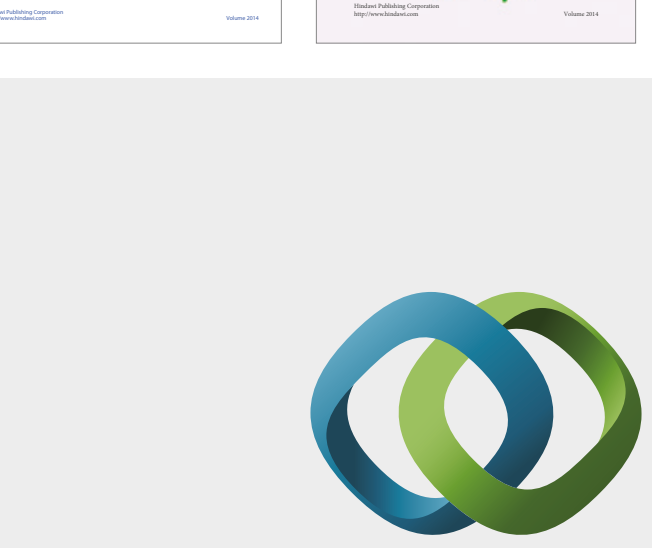

\section{Hindawi}

Submit your manuscripts at

https://www.hindawi.com
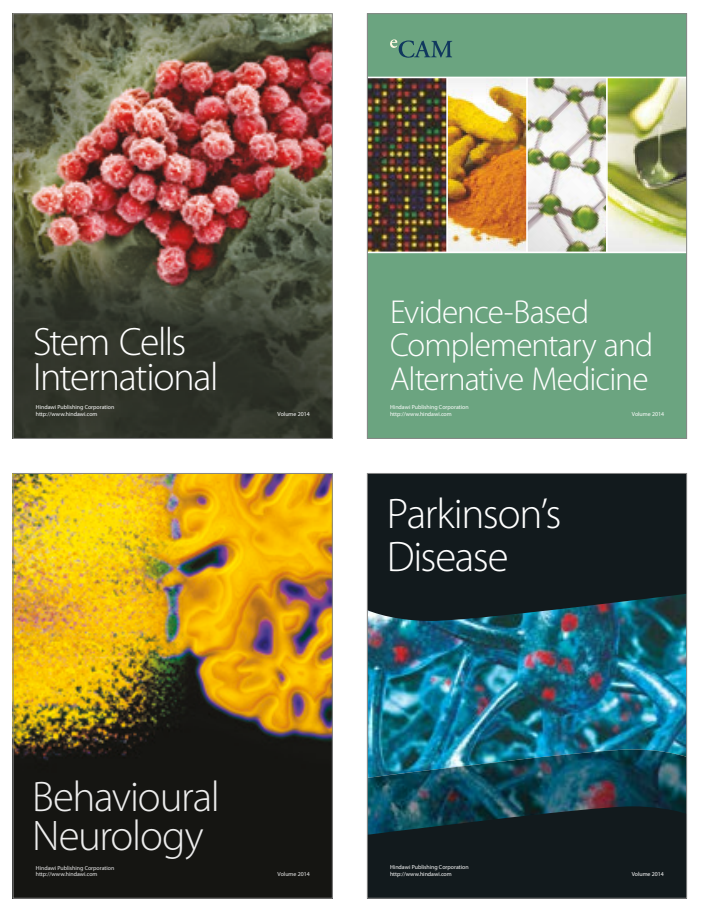
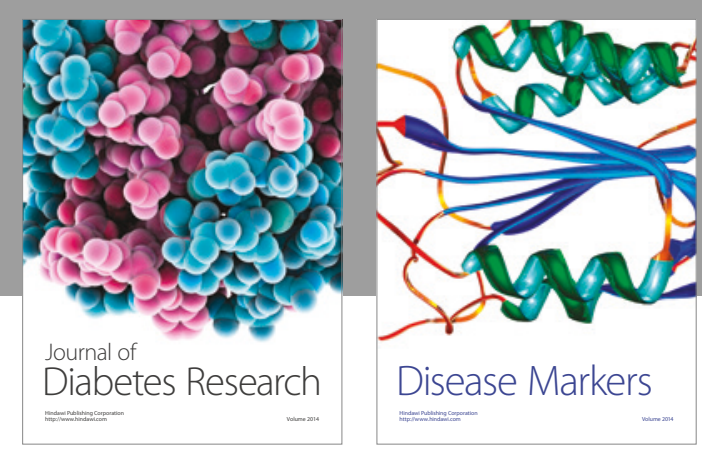

Disease Markers
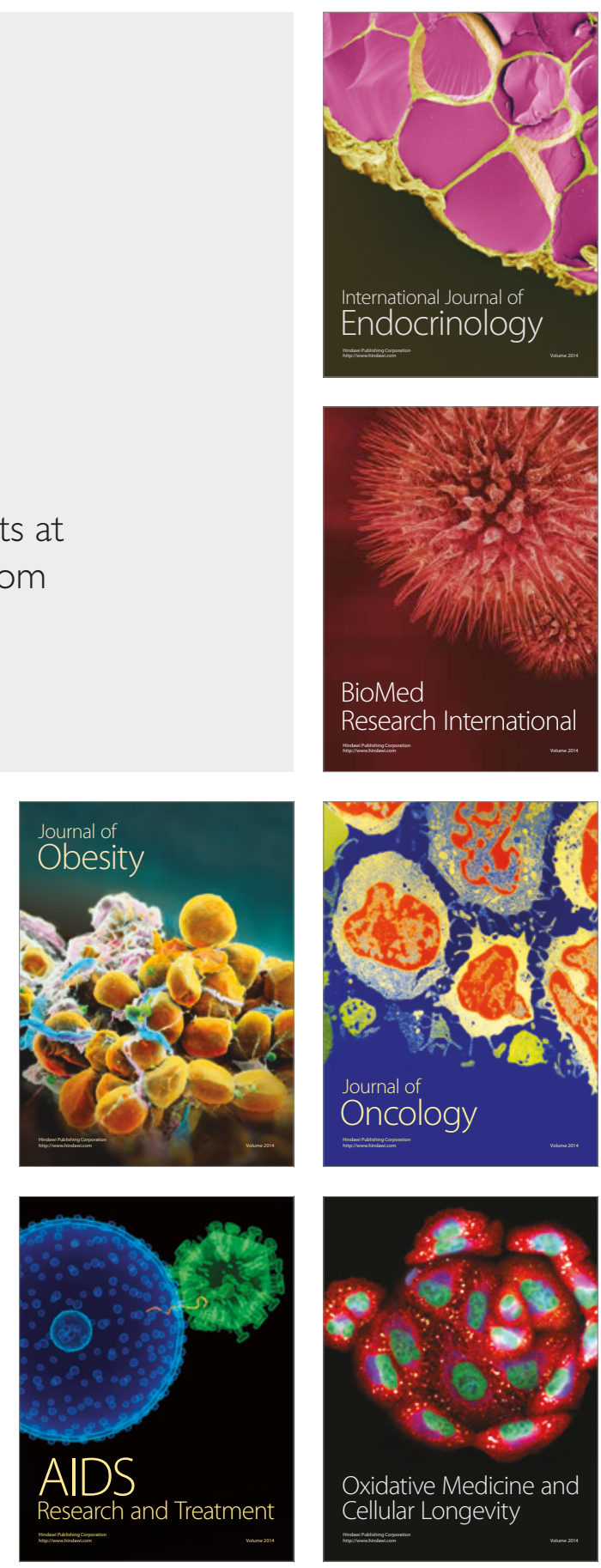